\title{
White Blister Rust Caused by Albugo candida on Oilseed Rape in Korea
}

\author{
Young-Joon Choi ${ }^{1,2}$, Mi-Jeong Park ${ }^{1}$, Ji-Hyun Park ${ }^{1}$ and Hyeon-Dong Shin ${ }^{1 *}$ \\ ${ }^{1}$ Division of Environmental Science and Ecological Engineering, Korea University, Seoul 136-701, Korea \\ ${ }^{2}$ Department of Organismic and Evolutionary Biology, Harvard University, Cambridge, MA 02138, USA
}

(Received on May 21, 2010; Accepted on July 9, 2010)

Oilseed rape, Brassica napus ssp. oleifera (DC.) Metzger, is grown worldwide for the production of vegetable oil for human consumption, animal feed, and biodiesel. In Korea, this crop has also been used as an important vegetable by harvesting the leaves in late winter or early spring or by picking the main flowering shoots just before the first flowers open. Since May 2004, typical symptoms of white blister rust disease on oilseed rape have been continuously found in several localities of Korea. Leaves of infected plants had whitish sori mostly on the lower surfaces and reddish violet blotches on the corresponding upper leaf surfaces (Fig. 1A-C). Representative samples were deposited in the herbarium (KUS-F20204, F22717, F24893).

Microscopic examination of the sori from fresh materials was performed under a DIC-light microscope (Zeiss AX10). The grouped sporangiophores were hyaline, clavate or cylindric, and measured 20-40 $\times 12-15 \mu \mathrm{m}$ (Fig. 1D \& E). The sporangia were arranged in basipetal chains, hyaline, globose to subglobose, with uniform wall thickness and measured $15-22 \times 12-19 \mu \mathrm{m}$ (Fig. 1F). The primary sporangia were similar to the secondary sporangia, although the former exhibit a slightly thicker wall than the latter. No resting organs were observed. Up to now, the white blister rust pathogen on oilseed rape has been considered Albugo candida or A. cruciferarum (cf. Farr and Rossman, 2010). Albugo cruciferarum is regarded as a synonym of $A$. candida (cf. Choi et al., 2007). Based on the morphological characteristics and the specific host plant, the causal agent of this disease was identified as $A$. candida (Pers.) Kuntze (Choi et al., 2007).

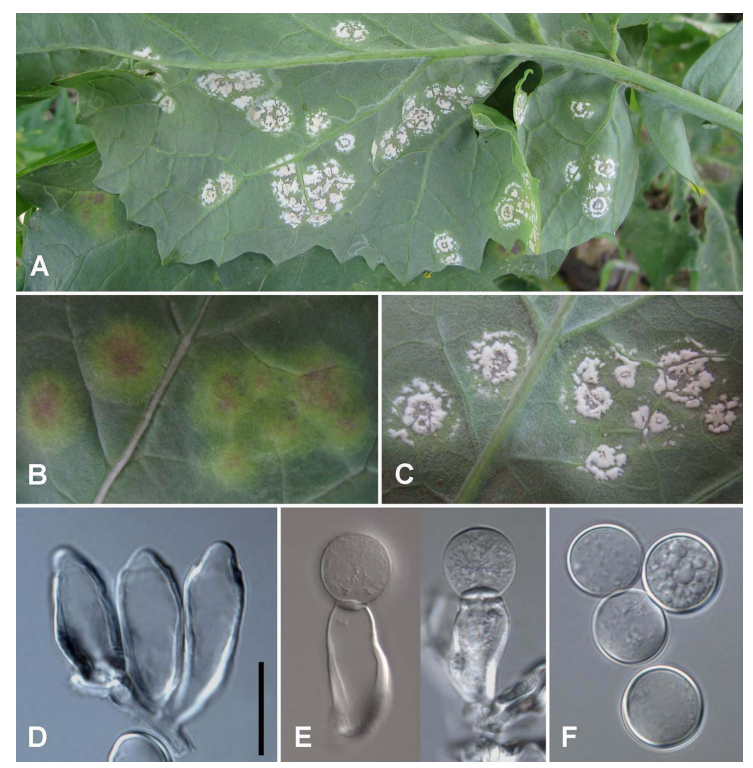

Fig. 1. White blister rust of oilseed rape associated with Albugo candida. (A) A typical symptom of white blister rust on a leaf. Close-up of reddish violet discolorations on the upper surface (B) and concentric sori on the lower surface (C). Sporangiophores without sporangium (D) and with sporangium (E), and also sporangia $(\mathrm{F})$ of $A$. candida. Scale bar $=20 \mu \mathrm{m}$.
The amplification and sequencing of $\operatorname{cox} 2 \mathrm{mtDNA}$ and ITS rDNA were performed according to a method of Choi et al. (2006), and the resulting sequences were deposited in GenBank (Acc. No. DQ409817 and DQ409816, respectively). In cox2 mtDNA-based phylogenetic analysis with Albugo s.str. sequences from GenBank (Fig. 2), the present causal agent formed a well-supported group with A. candida from Brassica juncea (AY927046) as well as various brassicaceous plants, among which no sequence difference was found. The sequence of ITS region was also identical to those of $A$. candida from B. oleracea (AF241767) and B. juncea (AY929828). Therefore, the sequence analysis verified the pathogen to be $A$. candida.

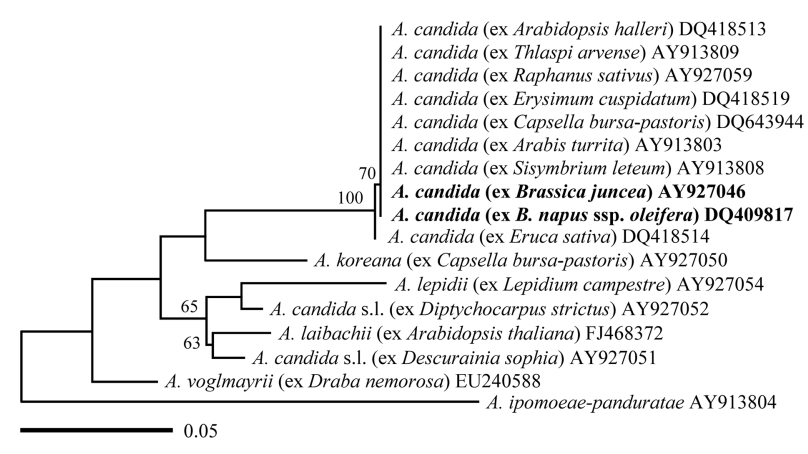

Fig. 2. Phylogenetic tree of Albugo s.str. inferred by Minimum Evolution analysis using the partial cox $2 \mathrm{mtDNA}$. Bootstrapping values above $50 \%$ are given above the branches. The number of nucleotide changes between taxa is represented by branch length and the scale bar equals the number of nucleotide substitutions per site. Albugo specimens from Brassica spp. are in bold.

In Korea, it has been reported that $A$. candida attacks $B$. juncea and $B$. campestris subsp. pekinensis (Korean Society of Plant Pathology, 2009), but to our knowledge this is the first record of $A$. candida on oilseed rape. As the white blister rust caused by $A$. candida is one of the most devastating diseases of oilseed rape in many countries where the crop is cultivated, the occurrence of this disease poses a significant threat for the commercial large-scale cultivation of oilseed rape in Korea.

\section{References}

Choi, Y. J., Hong, S. B. and Shin, H. D. 2006. Genetic diversity within the Albugo candida complex (Peronosporales, Oomycota) inferred from phylogenetic analysis of ITS rDNA and COX2 mtDNA sequences. Mol. Phylogenet. Evol. 40:400-409.

Choi, Y. J., Shin, H. D., Hong, S. B. and Thines, M. 2007. Morphological and molecular discrimination among Albugo candida materials infecting Capsella bursa-pastoris world-wide. Fungal Divers. 27:11-34.

Farr, D. F. and Rossman, A. Y. 2010. Fungal Databases, Systematic Mycology and Microbiology Laboratory, ARS, USDA. Retrieved May 18, 2010, from http://nt.ars-grin.gov/fungaldatabases/

Korean Society of Plant Pathology. 2009. List of Plant Diseases in Korea. 5 th ed., Suwon, Korea.

*Corresponding author (hdshin@korea.ac.kr) 